\title{
Mitotic phosphorylation of PRC1 at Thr470 is required for PRC1 oligomerization and proper central spindle organization
}

\author{
Chuanhai $\mathrm{Fu}^{1,2, *}$, Feng Yan ${ }^{1, *}$, Fang $\mathrm{Wu}^{1}$, Quan $\mathrm{Wu}^{1}$, Joseph Whittaker ${ }^{3}$, Haiying $\mathrm{Hu}^{4}$, Renming $\mathrm{Hu}^{4}$, \\ Xuebiao Yao $^{1,2}$ \\ ${ }^{1}$ Laboratory of Cellular Dynamics, Hefei National Laboratory and the University of Science and Technology of China, Hefei 230027, \\ China: ${ }^{2}$ Department of Physiology; ${ }^{3}$ Department of Anatomy \& Neurobiology, Morehouse School of Medicine, Atlanta, GA 30310, \\ USA; ${ }^{4}$ Department of Endocrinology, Huashan Hospital, Fudan University School of Medicine, Shanghai 200040, China
}

During cell division, chromosome segregation is orchestrated by the interaction of spindle microtubules with the centromere. A dramatic remodeling of interpolar microtubules into an organized central spindle between the separating chromatids is required for the initiation and execution of cytokinesis. Central spindle organization requires mitotic kinesins, the chromosomal passenger protein complex, and microtubule bundling protein PRC1. PRC1 is phosphorylated by Cdc2 at Thr470 and Thr481 during mitosis. However, the functional relevance of PRC1 phosphorylation at Thr470 has remained elusive. Here we show that expression of the non-phosphorylatable mutant $\mathrm{PRC1} 1^{\mathrm{T} 470 \mathrm{~A}}$ but not the phospho-mimicking mutant $\mathrm{PRC} 1^{\mathrm{T} 470 \mathrm{E}}$ causes aberrant organization of the central spindle. Immunoprecipitation experiment indicates that both $\mathrm{PRC} 1^{\mathrm{T} 470 \mathrm{~A}}$ and $\mathrm{PRC} 1^{\mathrm{T} 470 \mathrm{E}}$ mutant proteins associate with wild-type $\mathrm{PRC} 1$, suggesting that phosphorylation of Thr470 does not alter PRC1 self-association. In addition, in vitro co-sedimentation experiment showed that PRC1 binds to microtubule independent of the phosphorylation state of Thr470. Gel-filtration experiment suggested that phosphorylation of Thr470 promotes oligomerization of PRC1. Given the fact that prevention of the Thr470 phosphorylation inhibits PRC1 oligomerization in vitro and causes an aberrant organization of central spindle in vivo, we propose that this phosphorylation-dependent PRC1 oligomerization ensures that central spindle assembly occurs at the appropriate time in the cell cycle.

Keywords: central spindle, microtubule, oligomerization, PRC1, phosphorylation

Cell Research (2007) 17:449-457. doi: 10.1038/cr.2007.32; published online 17 April 2007

\section{Introduction}

The inheritance of the human genome through mitosis is precisely regulated by a series of tightly orchestrated events such as chromosome condensation, bi-orientated spindle formation, chromosome congression, segregation and cytokinesis. Cytokinesis plays an important role in

\footnotetext{
*These two authors contributed equally to this work Correspondence: Xuebiao Yao

Tel: +86-551-3606304; Fax: +86-551-3607141

E-mail: yaoxb@ustc.edu.cn

Received 4 September 2006; revised 19 January 2007; accepted 4 February 2007; published online 17 April 2007
}

the final stages of mitosis. The initiation of cytokinesis is always associated with the formation of a central spindle, a compacted and well-organized structure of bundled antiparalleled microtubules in the middle of two daughter cells. Perturbation of interactions between important components within the central spindle resulted in abnormalities of spindle microtubule organization, severe defects of membrane abscission and subsequent generation of bi-nucleated or multi-nucleated cells. Many proteins, such as MKLP1 [1], MKLP2 [2], KIF4 [3, 4], KIF14 [5], Aurora-B complex $[1]$ and PRC1 $[4,6]$, are involved in maintaining central spindle organization by orchestrating the segregation of sister chromatids and cytokinesis.

Among central spindle maintenance components, only 
two have been reported to mediate microtubule bundling in central spindle. One is centralspindlin, a hetero-tetramer containing CeMKLP1/ZEN-1 and RhoGAP/CYK-1 [7], while the other is an evolutionarily conserved protein, PRC1 (also named Feo in fruit fry, Ase1 in yeast and MAP65 in plant cells). PRC1 is a non-motor microtubule binding and bundling protein in human cells originally identified as a Cdc2 substrate essential for cytokinesis [6, 8]. Similar microtubule regulatory activities have been reported for its orthologs in yeast, fruit fly and plant . It is well known that overexpression of wild-type PRC1 in HeLa cells can result in a thick microtubule bundle at interphase [6]. Bundling activity of PRC1, as well as centralspindlin, is required for the organization of central spindle as well as for the successful progression of cytokinesis. PRC1 molecules accumulate on the midline of a central spindle with the cell cycle progression to anaphase. As a non-motor microtubule binding protein, transportation of PRC1 to the midline is promoted by its association with kinesin, KIF4, and timing of this progression is controlled through the dephosphorylation of Thr481 on PRC1 by the phosphatase Cdc14 when the cell exits metaphase [3, 8]. MKLP1, MKLP2, CENP-E, KIF14 and MgcRacGAP are also reported to interact with PRC1 [4, 5, 9]. Interestingly, the domains responsible for the interaction with these proteins are all mapped to the $\mathrm{N}$ terminal coiled-coil enriched domain of PRC1. However, it has remained elusive as to how the interactions of these protein with PRC1 are coordinated in mitosis. Depletion of PRC 1 expression by RNA interference totally disrupted the organization of central spindle in HeLa cells [4, 10], indicating that PRC1 is essential for the initiation of central spindle organization. However, the molecular mechanism underlying mitotic regulation of PRC1 in central spindle microtubule organization is not well understood. PRC1 contains two Cdc2 phosphorylation motifs located at Thr470 and Thr481 [8]. Using phospho-mimicking and non-phosphorylatable mutants, Mollinari and colleagues reported that phosphorylation at these two sites negatively regulates the microtubule bundling during early mitosis as overexpression of the non-phosphorylatable PRC1 (T470A and T481A) mutant causes extensive bundling of the prometaphase spindle [6]. Using a specific antibody against phosphorylated $\mathrm{Thr} 481$ and sucrose gradient fractionation, Zhu et al. [11] concluded that dephosphorylation of Thr481 is correlated with the oligomerization of PRC1 during metaphase-to-anaphase transition. However, it remains unclear whether phosphorylation of Thr470 plays any role in central spindle organization during the transition as most mutational analyses performed so far involved double mutations at both Thr470 and Thr481.

To evaluate the role of Thr470 phosphorylation in regulating central spindle organization, we carried out biochemical characterizations of recombinant PRC1 proteins including wild type, phospho-mimicking and nonphosphorylatable mutants. Our studies demonstrated that phosphorylation of Thr470 is essential for oligomerization of PRC1 in vitro. In addition, the non-phosphorylatable mutant PRC1 $1^{\mathrm{T} 470 \mathrm{~A}}$ leads to inhibition of PRC1 oligomerization in vitro and its overexpression causes an aberrant organization of central spindle in vivo.

\section{Materials and Methods}

\section{Cloning, mutagenesis and protein expression}

Full-length PRC1 cDNA was amplified by PCR and separately inserted into the pEGFP-C2 (Clontech), pET-28a (Novagen), pGEX6P-1 (Amersham Pharmacia) and p3XFLAG-myc-CMV ${ }^{\mathrm{TM}}-24$ vector (Sigma Chemicals, Inc.) at EcoR1 and Sal1 sites; a deletion mutant (304-620aa) was generated by PCR and was cloned into pEGFP-C2 (Clontech), pET-28a, PGEX-6P-1 vector, respectively. Two sitedirected mutants (T470A and T470E) were generated by the PCR amplification method using a specific mutation primer with $E c o$ R 1 and Sal1 restriction sites, then ligated into $\mathrm{pEGFP}-\mathrm{C} 2$ and $\mathrm{pET}-28 \mathrm{a}$ vector, respectively. All constructs were verified by DNA sequencing.

To directly observe the interaction between PRC1 proteins with preassembled microtubules in vitro, a new vector containing GFP and His tags were generated by inserting a GFP sequence cut from pEGFP-C2 into PET-28a wild type, deletion and single amino-acid mutants were cloned into this new vector for production of double tagged fluorescent proteins.

\section{Recombinant protein expression in bacteria}

Recombinant PRC1 and its mutants were expressed in bacteria as $6 \times$ histidine fusion proteins. Briefly, one liter of $\mathrm{LB}(10 \mathrm{~g} \mathrm{NaCl}$, $10 \mathrm{~g}$ tryptone and $5 \mathrm{~g}$ yeast extract, $\mathrm{pH}$ 7.4) was inoculated with bacteria transformed with histidine-PRC1, histidine-PRC1 ${ }^{\mathrm{T} 470 \mathrm{~A}}$, histidine-PRC1 $1^{\mathrm{T} 470 \mathrm{E}}$ or deletion mutants. When the optical density $\left(A_{600}\right)$ of the bacteria culture reached between 0.7 and 1.0 , expression of protein was induced by addition of $0.5 \mathrm{mM}$ isopropyl $\beta$-D-thiogalactopyranoside. Bacteria were harvested by centrifugation $3 \mathrm{~h}$ after induction, resuspended in PBS containing $5 \mu \mathrm{g} / \mathrm{ml}$ proteinase inhibitors (leupeptin, pepstatin and chymostatin), and sonicated for four bursts of $10 \mathrm{~s}$ each using a probe-tip sonicator. The lys ate was cleared of insoluble material by centrifugation at $10000 \times g$ for 20 min. The soluble fraction was applied to a column packed with Nickel-agarose beads (Qiagen), followed by extensive washes with PBS $(20 \times$ bead volume) containing $20 \mathrm{mM}$ imidazole. The fusion proteins on the beads were eluted in PBS containing $40 \mathrm{mM}$ imidazole and then applied to a gel filtration column (Econ-Pac 10 DG; Bio-Rad) to remove imidazole and exchange buffer for microtubule co-sedimentation assays. The fusion protein was estimated to be $90 \%$ pure by SDS-PAGE; major contaminants were degraded fragments of PRC1. Protein concentrations were determined by Bradford assay.

\section{Cell culture and transfection}

HeLa cells, from American Type Culture Collection (Rockville, MD, USA), were maintained as subconfluent monolayers in DMEM (Invitrogen, Carlsbad, CA, USA) with 10\% FBS (Hyclone, UT, USA) and $100 \mathrm{U} / \mathrm{ml}$ penicillin plus $100 \mu \mathrm{g} / \mathrm{ml}$ streptomycin (Invitrogen, Carlsbad, CA, USA). Plasmids were transfected into HeLa cells by 
using lipofectamine 2000 (Invitrogen; Carlsbad, CA, USA) according to the manufacturer's manual.

\section{Immunoprecipitation and immunoblotting}

Co-transfected HeLa cells were collected in PBS, and cell extracts were prepared in Tris buffer containing $50 \mathrm{mM}$ Tris- $\mathrm{HCl}(\mathrm{pH}$ 6.9), $150 \mathrm{mM} \mathrm{NaCl}, 2 \mathrm{mM}$ EDTA, $0.5 \%$ Triton X-100 plus protease inhibitor cocktail (leupeptin, pepstatin and chymostatin; $5 \mu \mathrm{g} / \mathrm{ml}$ ) and $1 \mathrm{mM}$ PMSF. Lysates were clarified and supernatants were then incubated with anti-FLAG antibody affinity beads (Sigma) for $4 \mathrm{~h}$ at $4{ }^{\circ} \mathrm{C}$. Beads were washed three times with the Tris buffer plus one time with PBS followed by boiling in SDS-PAGE sample buffer for $2 \mathrm{~min}$. After fractionation on SDS-PAGE, proteins were transferred to nitrocellulose membranes and probed with antibodies against the GFP epitope and FLAG, respectively. Immunoreactive signals were detected with ECL kit (Pierce) and visualized by autoradiography on Kodak BioMax film. Cells transfected with GFP-tagged proteins for expression testing were directly boiled in SDS-PAGE sample buffer for $5 \mathrm{~min}$, and samples were analyzed by immunoblotting with an anti-GFP antibody (Clontech) and an anti-PRC rabbit antibody [3].

\section{Immunofluorescence microscopy}

Cells were cultured on acid-treated glass coverslips. After transfection or drug treatment, cells were washed twice with PBS and once with PHEM (60 mM PIPES, 25 mM HEPES, 10 mM EGTA, $2 \mathrm{mM} \mathrm{MgCl}_{2}, \mathrm{pH}$ 6.9). Permeabilization was carried out for $1 \mathrm{~min}$ in PHEM buffer containing $0.1 \%$ Triton X-100 at room temperature. Pre-extracted cells were fixed in 3.7\% paraformaldehyde in PHEM buffer for $10 \mathrm{~min}$. After washing three times with PBS, cells were blocked with $1 \%$ BSA (Sigma) in PBS containing $0.05 \%$ tween- 20 for $30 \mathrm{~min}$, and then incubated with primary antibodies for $1 \mathrm{~h}$ followed by secondary antibodies for $30 \mathrm{~min}$. Slides were examined under a Zeiss Axiovert-200 fluorescence microscope, and images were collected and processed with Adobe Photoshop 7.0 software.

\section{Tubulin purification, microtubule binding and bundling as- say}

Tubulin was purified from porcine brains with traditional two cycles of temperature-dependent polymerization/depolymerization method. The purified depolymerized tubulin was further purified on phosphocellulose column (P11, Whatman) to remove MAP (microtubule associated proteins).

Reconstituted microtubules were made by incubation of $2 \mu \mathrm{g} / \mu \mathrm{l}$ purified tubulin in PEM Buffer $(80 \mathrm{mM}$ Pipes, $1 \mathrm{mM}$ EGTA, $1 \mathrm{mM}$ $\mathrm{MgCl}_{2}, \mathrm{pH}$ 6.8) containing $1 \mathrm{mM} \mathrm{GTP}$ for $20 \mathrm{~min}$ at $37^{\circ} \mathrm{C}$. Purified His-tagged proteins were added to the above reconstituted microtubules and incubated for additional $15 \mathrm{~min}$ incubation at $32{ }^{\circ} \mathrm{C}$. Then, the microtubule and protein solution were clarified by ultracentrifugation (90 000×g, Beckman, TLA-100). Supernatant and pellet of the ultracentrifugation were subjected to SDS-PAGE fractionation and visualized by Coomassie blue staining.

To visualize how PRC1 proteins (wild type, non-phosphorylatable and phosphor-mimicking mutants) interact with microtubules, rhodamine-conjugated tubulin (Cytoskeleton Inc.) was preassembled with taxol (Sigma). Briefly, aliquots of $9 \mu$ l rhodamine-conjugated tubulin $(5 \mu \mathrm{g} / \mu \mathrm{l})$ were mixed with $1 \mu \mathrm{l}$ taxol $(20 \mu \mathrm{M}$ in DMSO) and incubated at $37{ }^{\circ} \mathrm{C}$ for $20 \mathrm{~min}$. The taxol-stabilized microtubules were then incubated with GFP- and His-tagged PRC1 proteins (5 $\mu \mathrm{g} / \mu \mathrm{l})$. After $15 \mathrm{~min}$ incubation at $32{ }^{\circ} \mathrm{C}$, the mixtures were fixed by $1 \%$ glutaradehyde in PEM buffer, then squashed onto $18 \times 18 \mathrm{~mm}$ coverslips as previously described [12]. The coverslips were then mounted onto slides and examined under a fluorescent microscope (Axiovert-200, Carl Zeiss, Germany).

\section{Gel filtration chromatography}

In general, approximately $2 \mathrm{mg}$ His-tagged proteins were concentrated to $2 \mathrm{ml}$, and then injected into Superdex 200 column (Amersham Pharmacia) equipped with FPLC apparatus (Amersham Pharmacia), followed by collection of $1-\mathrm{ml}$ fractions. The protein concentration in each individual fraction was measured by absorbance at $280 \mathrm{~nm}$ using a spectrometer.

\section{Results}

Phosphorylation of PRC1 at T470 is essential for the organization of central spindle

PRC1 was originally identified as a Cdc2 substrate located to the midzone and essential for cytokinesis [8]. $\mathrm{PRC} 1$ has an N-terminal oligomerization domain, a central MT-binding domain, and two Cdc2 phosphorylation sites at the junction between the MT-binding domain and the Cterminal region $[6,8]$. Previous study has mapped the $\mathrm{Cdc} 2$ phosphorylation sites to Thr470 and Thr481 as illustrated in Figure 1A, and it has been reported that a Cdc2-nonphosphorylatable mutant of PRC $1^{\text {T481A }}$ causes extensive bundling of the metaphase spindle $[6,8]$. To elucidate the functional relevance of Thr470 phosphorylation in mitosis, we transfected wild-type PRC1, phospho-mimicking mutant $\mathrm{PRC} 1^{\mathrm{T} 470 \mathrm{E}}$ and non-phosphorylatable mutant $\mathrm{PRC} 1^{\mathrm{T} 470 \mathrm{~A}}$ into asynchronized HeLa cells. The transfected cells were harvested at $36 \mathrm{~h}$ after the transfection for analyzing the expression of GFP-PRC1 proteins. Western blotting analysis showed that wild type and mutant GFP-PRC1 proteins migrated at $\sim 100 \mathrm{kDa}$, an expected size for the fusion proteins (Figure 1B). Western blotting analysis using a PRC1 antibody showed that the expression level of GFP-PRC1 is about four-fold higher than that of endogenous protein in positively transfected cells (data not shown).

To explore whether Cdc2-mediated phosphorylation of PRC1 at Thr470 modulates the central spindle organization, constructs for GFP-tagged wild type, non-phosphorylatable and phospho-mimicking PRC1 were transfected into HeLa cells and fusion proteins were scored for their central spindle localization relative to midzonal microtubules under fluorescence microscopy. The transfected cells were triply scored for $\beta$-tubulin (red), GFP-PRC1 (green) and DNA (blue). Figure $1 \mathrm{C}$ shows optical sections from anaphase and telophase cells. Similar to what has been noted earlier [6, 8], exogenous wild-type GFP-PRC1 is localized to the central spindle as inter-digitations (Figure $1 \mathrm{C}, b$, arrow). The GFP-PRC1 labeling is overlapped with that of midzonal microtubules. As cell cycle progresses into late telophase, a portion of GFP-PRC1 becomes concentrated at the midbody (Figure 1C, $b^{\prime}$, arrowhead), while the majority distributes 
A

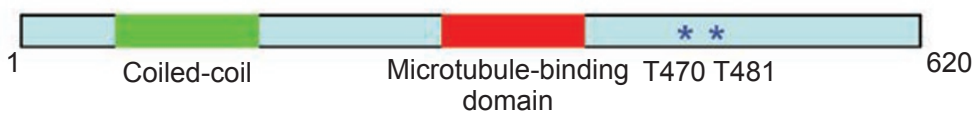

B

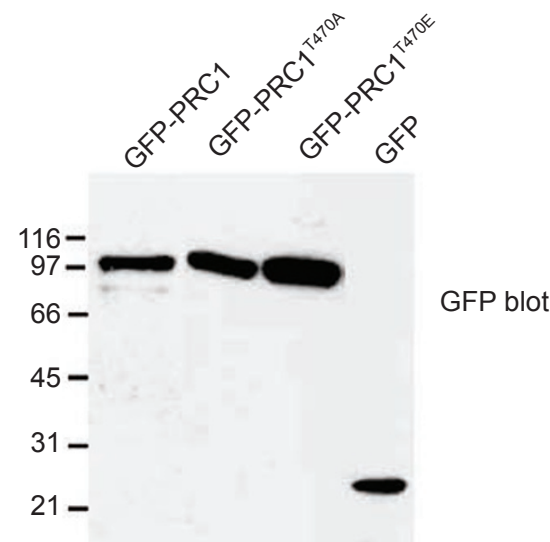

D

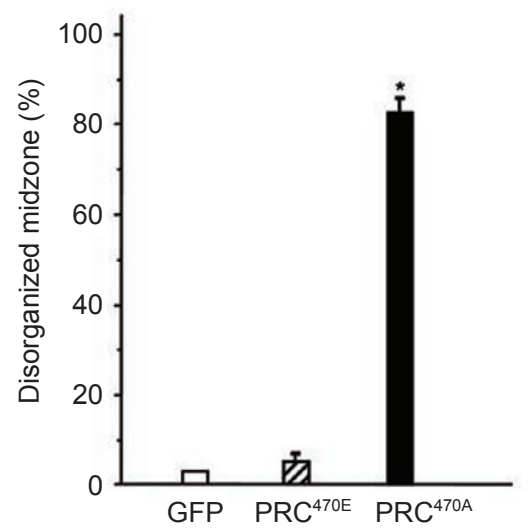

C

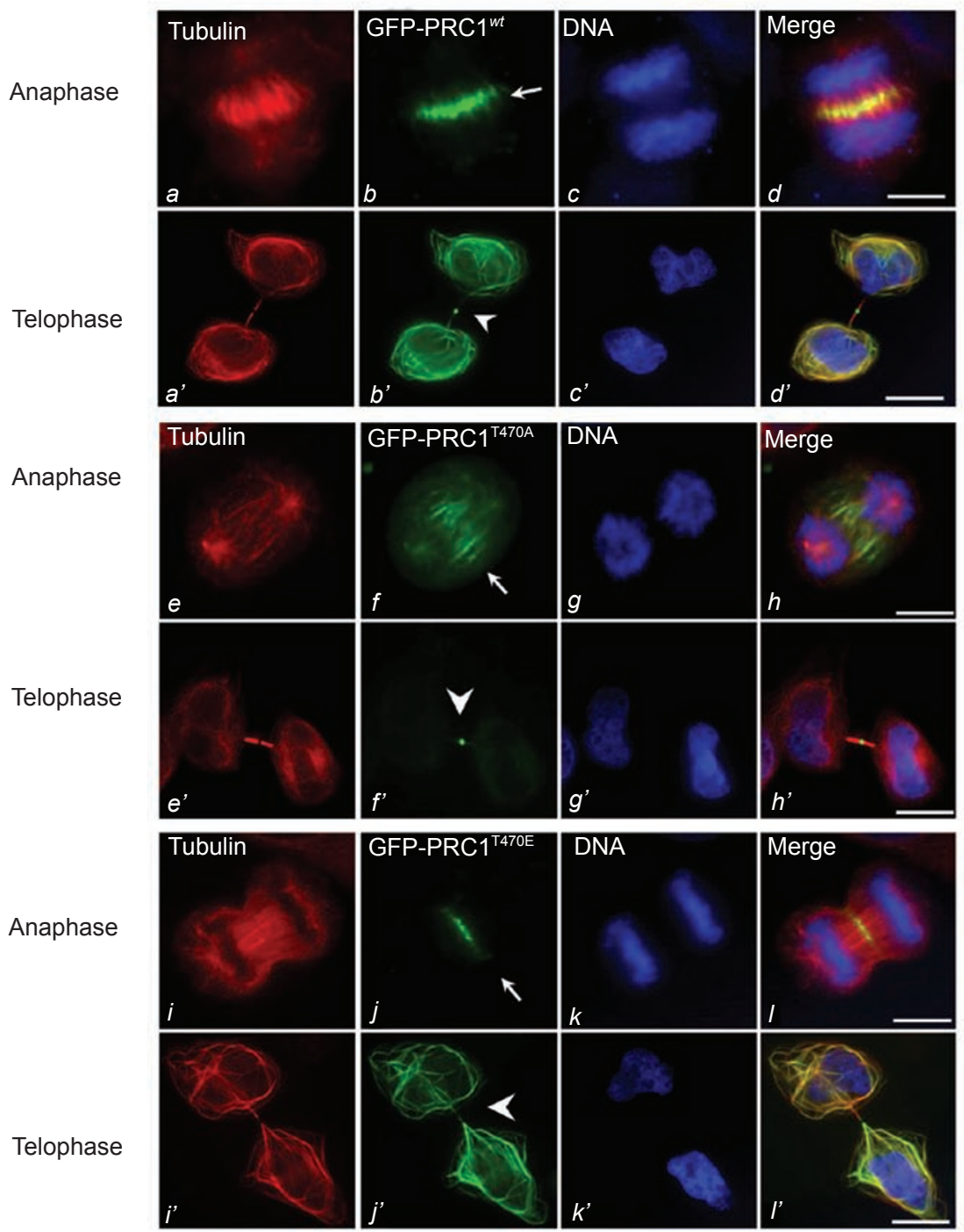


in the cytoplasm as filaments that are superimposed onto microtubules (Figure 1C, $d^{\prime}$ ). The distribution of $\mathrm{PRC} 1^{\mathrm{T} 470 \mathrm{E}}$ was similar to that of endogenous PRC1 (Figure 1C, $i-l$, $i^{\prime}-l$ '). However, the GFP-PRC $1^{\mathrm{T} 470 \mathrm{~A}}$-expressing anaphase cells contain many disorganized central spindles, which were readily observed (Figure $1 \mathrm{C}, f$, arrow). Interestingly, the GFP-PRC1 $1^{\mathrm{T} 470 \mathrm{~A}}$-expressing telophase cells contain little cytoplasmic filaments while the midbody localization of the GFP signal remains (Figure 1C, $f^{\prime}$, arrowhead). Our quantitative analysis from three separate experiments indicated that more than $80 \%$ of $\mathrm{PRC}^{\mathrm{T} 470 \mathrm{~A}}$-expressing anaphase cells display aberrant central spindle organization (Figure 1D). These data demonstrated that phosphorylation of PRC1 at Thr470 is important for proper organization of the central spindle in anaphase cells.

\section{Phosphorylation of T470 is not required for PRC1 self- association}

It has been proposed that PRC1 self- associates in vivo. To determine whether Cdc2-mediated phosphorylation of PRC1 at Thr470 modulates PRC1-PRC1 interaction, we used anti-FLAG antibody to isolate FLAG-PRC1 from lysates of HeLa cells transiently transfected to co-express FLAG-PRC1 with GFP-PRC1, or GFP-PRC1 ${ }^{\text {T470A }}$, GFP$\mathrm{PRC1}^{\mathrm{T} 470 \mathrm{E}}$ and GFP-histone $2 \mathrm{~B}$, respectively. Silver staining clearly showed that GFP-tagged full-length PRC1 and the two mutants but not Histone H2B co-precipitated well with FLAG-PRC1 (Figure 2A). Western blot analysis of proteins precipitated by FLAG antibody beads revealed that both wild-type PRC1 and the mutant $\mathrm{PRC1}^{\mathrm{T} 470 \mathrm{~A}}$ and $\mathrm{PRC}^{\mathrm{T}}{ }^{\mathrm{T} 70 \mathrm{E}}$ proteins were retained by FLAG-PRC1 (Figure 2B), indicating that phosphorylation of Thr470 does not modulate PRC1-PRC1 self-association. Although the two mutants of PRC1 can bind to FLAG-PRC1, PRC1 ${ }^{\text {T470E }}$ exhibited a stronger binding than PRC1 ${ }^{\mathrm{T} 470 \mathrm{~A}}$. Nevertheless, phosphorylation of Thr470 is not required for the PRC1PRC1 intermolecular interaction.

\section{Phosphorylation of T470 does not modulate the binding of PRC1 to microtubule}

Previous studies showed that expression of the nonphosphorylatable PRC1 $1^{\mathrm{T} 470 \mathrm{~A} / \mathrm{T} 481 \mathrm{~A}}$ mutant caused extensive bundling of the spindle microtubules and the central spindle microtubules in mitotic cells [6]. Since Thr470 is located around the microtubule-binding domain, we decided to examine whether phosphorylation of $\mathrm{Thr} 470$ modulates PRC1-microtubule interactions.

To determine whether PRC1 stably binds to microtubules in a phosphorylation-regulated manner, we assayed the co-sedimentation of PRC1 with microtubule. Recombinant wild-type PRC1, and the mutant $\mathrm{PRC} 1^{\mathrm{T} 470 \mathrm{~A}}$ and $\mathrm{PRC} 1^{\mathrm{T} 470 \mathrm{E}}$ proteins were incubated with microtubules under polymerizing conditions for $20 \mathrm{~min}$ at $32{ }^{\circ} \mathrm{C}$, and the microtubules were pelleted by centrifugation at $90000 \times \mathrm{g}$ for $15 \mathrm{~min}$. Microtubule polymers sediment under these conditions. The Coomassie blue-stained gel in Figure 3A shows that wild-type PRC1 was obviously detected in the pellet when microtubules were polymerized. Examination of recombinant $\mathrm{PRC} 1^{\mathrm{T} 470 \mathrm{~A}}$ and $\mathrm{PRC} 1^{\mathrm{T} 470 \mathrm{E}}$ proteins revealed that they also both co-sedimented with microtubules. It is also apparent that phosphorylation of Thr470 did not dramatically affect the amount of tubulin that was sedimented.

We also assayed the co-sedimentation of PRC1 C-terminal portion with microtubule given that the predicted microtubule-binding domain is near Thr470 (Figure 3B). Recombinant C-terminal portion of PRC1 $(\mathrm{PRC} 1 \Delta \mathrm{N})$ or the corresponding mutant $\mathrm{PRC} 1 \Delta \mathrm{N}^{\mathrm{T} 470 \mathrm{~A}}$ and $\mathrm{PRC} 1 \Delta \mathrm{N}^{\mathrm{T} 470 \mathrm{E}}$ proteins were incubated with microtubules under polymer-

Figure 1 Phosphorylation of PRC1 at T470 is essential for faithful organization of the central spindle. (A) Schematic illustration of PRC1 structure and two Cdc2 phosphorylation sites. (B) Expression of exogenous GFP-PRC1 proteins in HeLa cells. HeLa cells transfected with GFP-PRC1 (wild type, T470A and T470E) were collected $36 \mathrm{~h}$ post-transfection as described in "Materials and Methods." Equal amount of protein samples $(35 \mu \mathrm{g})$ from both mock-transfected and GFP-PRC1-transfected cells were separated on SDS-PAGE, blotted to nitrocellulose and probed by an anti-GFP antibody followed by ECL detection. The GFP antibody recognizes exogenously expressed GFP-PRC1 proteins with a predicted size of $100 \mathrm{kDa}$. (C) Non-phosphorylatable mutant PRC1 T470A causes aberrant organization of central spindle. HeLa cells grown on coverslips were transfected with $\mathrm{pEGFP-PRC1,pEGFP-PRC1}{ }^{\mathrm{T} 470 \mathrm{~A}}$ and pEGFP-PRC1 ${ }^{\mathrm{T} 470 \mathrm{E}}$, respectively. Thirty-six hours after transfection, cells were fixed, permeabilized and stained for tubulin (red) and DAPI (DNA, blue). The two panels show representative HeLa cell staining in anaphase and telophase. ( $a$ - $d$ ) Wild-type GFP-PRC1 signal marks the midzone of anaphase cells ( $b$, green) which is overlapped with central spindle microtubule $(a$, red) as shown in the merge. PRC1 is co-distributed with microtubule at the midbody of telophase cells ( $d$ ', merge). Phospho-mimicking PRC1 mutant $\mathrm{PRC}^{\mathrm{T} 470 \mathrm{E}}$ displays a similar cellular distribution profile like that of wild type $\left(i-l\right.$ and $\left.i^{\prime}-l^{\prime}\right)$ ). However, the T470 mutant PRC1 (but not T470E) attenuates the organization of central spindle, suggesting that Cdc2-mediated phosphorylation of $\mathrm{Thr}^{\mathrm{T} 470} \mathrm{on} \mathrm{PRC1} \mathrm{is}^{\mathrm{P}}$ required for proper central spindle assembly. Bar $=5 \mu \mathrm{m}$. (D) Quantification of disorganized central spindle in GFP-PRC1-expressing cells. At least 30 cells containing GFP signal in anaphase from each of three independent experiments were examined. The error bars represent S.E.; $n=3$ preparations. ${ }^{*} p<0.01$. 

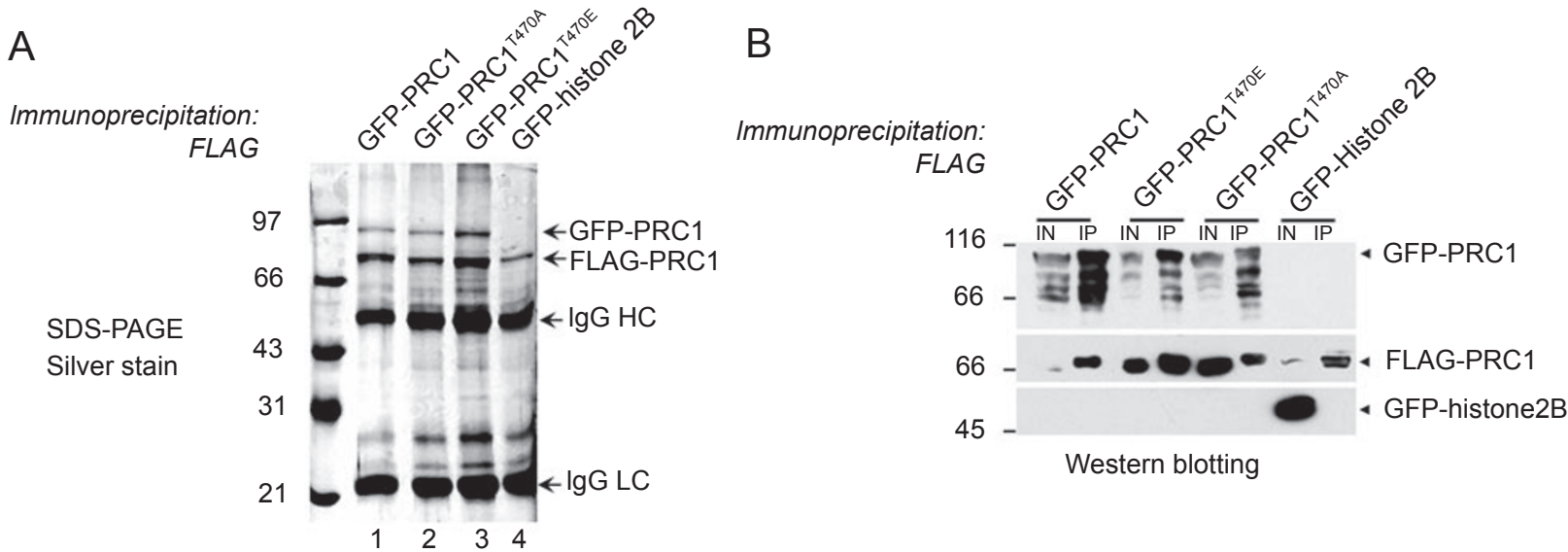

Figure 2 Phosphorylation of T470 is not required for inter-molecular association of PRC1. (A) Aliquots of extracts from 293 cells transiently co-transfected with FLAG-PRC1 and GFP-PRC1, GFP-PRC1 ${ }^{\text {T470A }}$, GFP-PRC1 ${ }^{\text {T470E }}$ or GFP-histone $2 \mathrm{~B}$ were incubated with $5 \mu \mathrm{l}$ anti-FLAG antibody beads. The immunoprecipitates were resolved by SDS-PAGE followed by silver staining. Immunoglobin heavy chain (IgG HC), light chain (IgG LC) and two prominent bands ( 73 and $\sim 100 \mathrm{kDa})$ were readily detected in the silver-stained gel. The $73 \mathrm{kDa}$ band is FLAG-PRC1 while the $100 \mathrm{kDa}$ band is GFP-PRC1. (B) Aliquots of extracts from 293 cells transiently co-transfected with FLAG-PRC1 and GFP-PRC1, GFP-PRC1 ${ }^{\text {T470A }}$, GFP-PRC1 ${ }^{\mathrm{T} 470 \mathrm{E}}$ or GFP-histone $2 \mathrm{~B}$ were incubated with $5 \mu \mathrm{l}$ anti-FLAG antibody-immobilized agarose beads as described in "Materials and Methods." The immunoprecipitates were resolved by SDS-PAGE followed by immunoblotting analyses with antibodies against GFP (GFP-PRC1; GFP-histone 2B) and FLAG (FLAG-PRC1). FLAG-PRC1 pulls down wild-type GFP-PRC1, GFP-PRC1 ${ }^{\text {T470A }}$ and GFP-PRC1 ${ }^{\text {T470E }}$ proteins but not GFP-histone 2B. IN: cell lysate input; IP: immunoprecipitates.

izing conditions for $20 \mathrm{~min}$ at $32{ }^{\circ} \mathrm{C}$, and the microtubules were pelleted by centrifugation at $90000 \times \mathrm{g}$ for $15 \mathrm{~min}$. The Coomassie blue-stained gel in Figure $3 \mathrm{C}$ shows that $\mathrm{PRC} 1 \Delta \mathrm{N}$, phospho-mutant $\mathrm{PRC} 1 \Delta \mathrm{N}^{\mathrm{T} 470 \mathrm{~A}}$ and $\mathrm{PRC} 1 \Delta \mathrm{N}^{\mathrm{T} 470 \mathrm{E}}$ proteins were all detected in the pellet when microtubules were polymerized. Thus, we conclude that phosphorylation of Thr 470 does not modulate the association of PRC1 with microtubules.

To visualize how recombinant PRC1 proteins bind to microtubules and whether they bundle microtubules, we generated recombinant GFP-PRC1 proteins that enable us to visualize the localization of PRC1 protein relative to pre-assembled microtubules and evaluate whether PRC1 protein modulate microtubule bundling under a microscope. As shown in Figure 3D, addition of the phospho-mimicking that $\mathrm{PRC} 1^{\mathrm{T} 470 \mathrm{E}}$ dramatically promoted the bundling of microtubules (h) while the non-phosphorylatable mutant $\mathrm{PRC}^{\mathrm{T}}{ }^{\mathrm{T} 70 \mathrm{~A}}$ binds along the microtubules but has no obvious bundling effect (e). Examination of pre-assembled microtubules incubated with his-tagged GFP did not reveal any labeling of GFP on the microtubules, suggesting that the binding of PRC1 proteins with microtubules is specific and independent of the GFP tag. Thus, we conclude that phosphorylation of Thr470 does not modulate the association of PRC1 with microtubules but stimulates the microtubule bundling.
Phosphorylation of Thr470 is required for oligomerization of PRC1

Previous studies indicated that Thr470 and Thr481 on PRC1 were the two phosphorylation sites for CDC2-cyclin, and dephosphorylation of phosphorylated Thr481 before anaphase was required for the transportation of PRC1 to the central spindle via the motor activity of KIF4 [3, 8]. During the preparation of this manuscript, Zhu et al. [11] showed that Thr481-unphosphorylated PRC1 forms oligomers (probably tetramers) via its N-terminal oligomerization domain in vivo. To illustrate the functional relevance of Thr470 phosphorylation on PRC1 oligomerization, recombinant wild-type PRC, and mutant PRC1 $1^{\mathrm{T} 470 \mathrm{~A}}$ and PRC1 ${ }^{\mathrm{T} 470 \mathrm{E}}$ proteins were purified to homogeneity and fractionated by gel-filtration. The eluation profile indicates that purified wild-type PRC1 is eluted as a single sharp peak, corresponding to a protein complex with an approximate molecular mass of $282 \mathrm{kDa}$. This result indicated that wildtype PRC1 forms tetramers in vitro (Figure 4A). $\mathrm{PRC1}^{\mathrm{T} 470 \mathrm{~A}}$ protein is eluted as a major peak corresponding to a protein complex with an approximate molecular mass of $141 \mathrm{kDa}$ and a shoulder peak corresponding to the molecular mass of $282 \mathrm{kDa}$, suggesting that the non-phosphorylatable $\mathrm{PRC}^{\mathrm{T} 470 \mathrm{~A}}$ mutant protein forms a mixture of dimers and tetramers in vitro (Figure 4B). However, the phosphomimicking mutant $\mathrm{PRC}^{\mathrm{T} 470 \mathrm{E}}$ protein is eluted as a major 
A

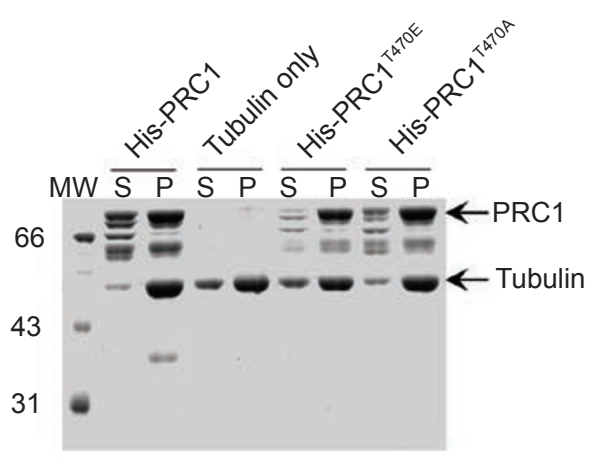

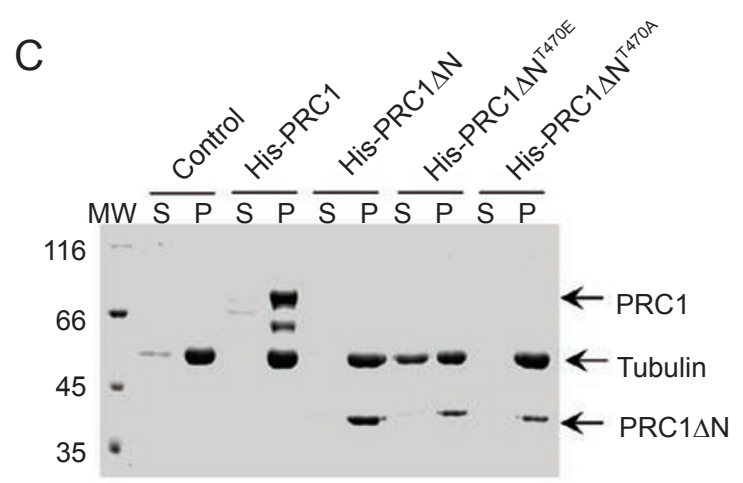

620

PRC1 620

$\mathrm{PRC} 1 \Delta \mathrm{N}$

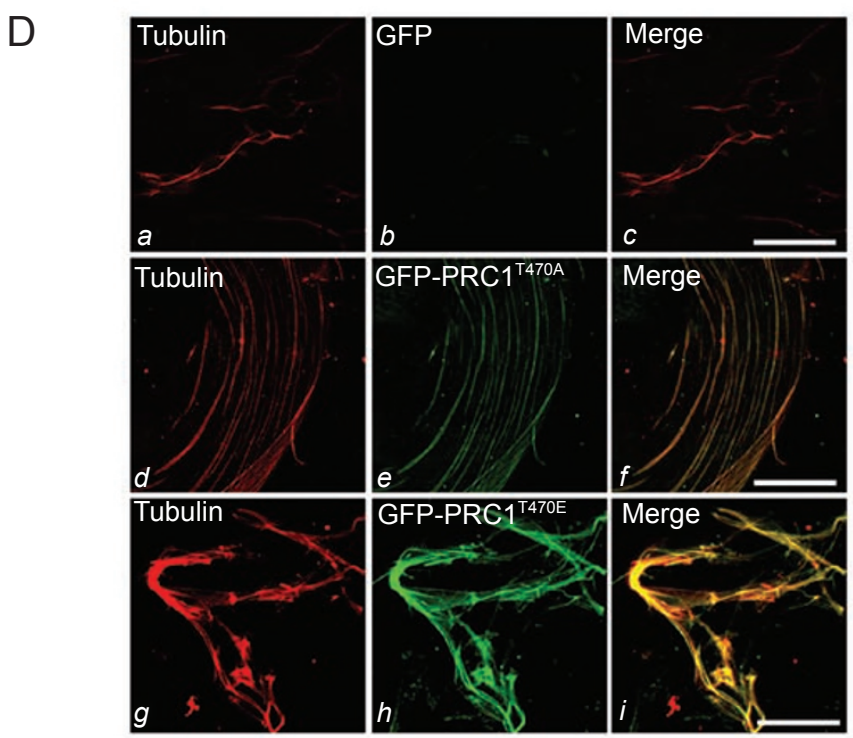

Figure 3 Phosphorylation of T470 does not modulate the binding of PRC1 to microtubule. (A) The purified bacterial expressed Histagged full-length PRC1 (indicated as His-PRC1) and the two mutants (His-PRC1 ${ }^{470 \mathrm{~A}}$ and His-PRC1 ${ }^{470 \mathrm{E}}$ ) were incubated with aliquots of tubulin in polymerization buffer at $32{ }^{\circ} \mathrm{C}$ for $20 \mathrm{~min}$ and centrifuged as described in "Materials and Methods." Equal volumes of supernatant $(S)$ and pellet $(P)$ fractions were resolved by electrophoresis, and proteins were visualized by Coomassie blue staining. An aliquot of tubulin, without addition of PRC1 protein, was included to examine the quality of tubulin polymerization. (B) Schematic illustration of the PRC1 N-terminal deletion mutant that contains the microtubule-binding domain and Cdc2 phosphorylation sites. (C) The purified recombinant full-length PRC1 with His-tag (indicated as His-PRC1), the mutant PRC1 $\Delta \mathrm{N}$ with the $\mathrm{N}$-terminal deletion, His-PRC $1 \Delta \mathrm{N}^{470 \mathrm{~A}}$ and His-PRC1 $1 \Delta \mathrm{N}^{470 \mathrm{E}}$ were incubated with aliquots of tubulin in polymerization buffer for 20 min and centrifuged as described in "Materials and Methods." Equal volumes of supernatant $(S)$ and pellet $(P)$ fractions were resolved by electrophoresis, and proteins were visualized by Coomassie blue staining. An aliquot of tubulin, without addition of PRC1 protein, was included to examine the quality of tubulin polymerization. (D) His-GFP-tagged PRC proteins were expressed and purified from bacteria as described in "Materials and Methods." The purified proteins (b, e, h) were incubated for 15 min with taxol-stabilized microtubules preassembled from rhodamine-tubulin $(\mathrm{a}, \mathrm{d}, \mathrm{g})$. The mixture was then deposited on top of a poly-lysine-treated coverslip, which was then fixed and examined under an inverted fluorescent microscope. Microtubules appear short and unbundled in the absence of His-PRC1 (a). After addition of His-PRC1 ${ }^{\mathrm{T} 470 \mathrm{E}}$, microtubles became long and highly bundled filaments while the incubation of microtubles with His- $\mathrm{PRC1} 1^{\mathrm{T} 470 \mathrm{~A}}$ resulted in long and highly organized but not bundled filaments. Bar $=40 \mu \mathrm{m}$. 

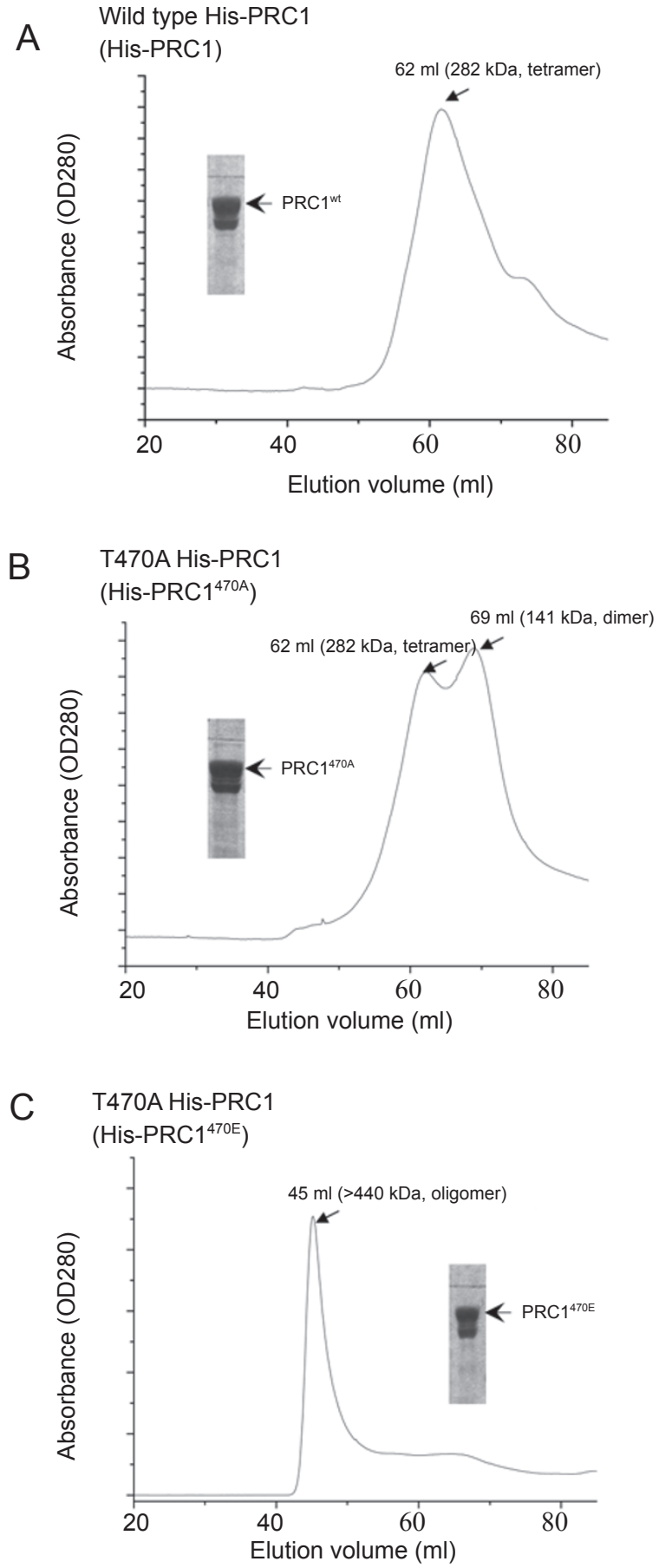

Figure 4 Phosphorylation of T470 promotes oligomerization of PRC1. Gel filtration analysis of His-PRC1 wild type (A), His$\mathrm{PRC}^{470 \mathrm{~A}}(\mathbf{B})$ and His-PRC $1^{470 \mathrm{E}}(\mathbf{C})$. The proteins were individually loaded onto Superdex 200 and separated by FPLC. Each profile of A280 vs elution volume was recorded. Ferritin (440 kDa), Catalase (232 kDa), Adolase (158 kDa), Albumin (67 kDa), Ovalbumin (43 kDa), Chymotrypsinogen A (25 kDa) and Ribonuclease A (13 kDa) were used as molecular weight markers for the calibration. Maximum UV absorbance of each analyzed protein was indicated by arrows and the corresponding elution volume was used to determine their molecular weight. peak corresponding to a protein complex with approximate molecular mass of $440 \mathrm{kDa}$, and this result indicated that the $\mathrm{PRC} 1^{\mathrm{T} 470 \mathrm{E}}$ mutant forms oligomers in vitro (Figure $4 \mathrm{C}$ ). Thus, phosphorylation of PRC1 at Thr470 is likely essential for oligomerization.

\section{Discussion}

We provide here the first evidence showing that inhibition of mitotic phosphorylation of PRC1 at Thr470 perturbs the organization of central spindle during mitosis. Our biochemical characterization demonstrates that phosphorylation of PRC1 at Thr470 is not essential for PRC1microtubule interaction, but is essential for the formation of PRC1 oligomers. We sought to pinpoint the regions of PRC1 which mediate the self-association in vitro. Our preliminary pull-down assay suggests that both the $\mathrm{C}$-terminal domain and the $\mathrm{N}$-terminal domain of PRC1 can mediate self-association (Fu et al., unpublished observation). Further characterization is required to delineate the molecular determinant(s) underlying PRC1 oligomerization and its regulation by Thr470 phosphorylation. Given the disorganization of central spindle seen in cells overexpressing the non-phosphorylatable $\mathrm{PRC1}^{\mathrm{T} 470 \mathrm{~A}}$ mutant, along with the inability of $\mathrm{PRC1}^{\mathrm{T} 470 \mathrm{~A}}$ protein to form oligomers in vitro, we propose that this Thr470 phosphorylation-dependent formation of PRC1 oligomers is essential for the initiation of central spindle organization in mitosis.

The spindle midzone, a highly organized network of anti-parallel interdigitating microtubules between separating chromosomes, plays a crucial role in regulating the initiation and completion of cytokinesis. Cytokinesis is a highly orchestrated process that must be coordinated precisely with other events of the cell cycle by coupling cytokinesis to $\mathrm{Cdc} 2$ inactivation. Downregulation of Cdc2 activity initiates the accumulation of some proteins on the midline of central spindle. These proteins include MKLP1, MKLP2, MgcRacGAP and PRC1, etc. The dephosphorylation of MKLP1 by an unknown phosphotase at anaphase releases the inhibition in MKLP1-mediated microtubule bundling $[13,14]$. Similarly, PRC1 is phosphorylated on Thr470 and Thr481 by Cdc 2 in early mitosis $[3,6$, 8]. The dephosphorylation of Thr481 is essential for the PRC1-KIF4 interactions required for transporting PRC1 to the midline of central spindle [3]. However, the spatiotemporal profiles concerning the phosphorylation and dephosphorylation of PRC1 at Thr481 and Thr470 are not well understood. In particular, little information is known for the regulation of Thr470 phosphorylation in mitosis. The fact that the inhibition of Thr481 phosphorylation by overexpression of the T481A mutant in HeLa cells does not interfere with the microtubule bundling of metaphase 
spindle [6] supports our finding that phosphorylation of Thr470 alone is sufficient to promote microtubule bundling (e.g., Figure 3D). The present finding that phosphorylation of PRC1 at Thr 470 promotes the formation of PRC1 oligomers provides novel insights for a better understanding of the spatiotemporal control of central spindle assembly. It would be of great interest to develop phospho-Thr470 specific antibody, so that the spatiotemporal order of PRC1 phosphorylation at $\mathrm{Thr} 470$ can be illustrated in comparison with that of Thr481. It has been shown that Cdc2-mediated phosphorylation of PRC1 at Thr481 controls the timing of association and translocation of PRC1 by KIF4 [3]. The availability of phospho-Thr470 specific antibody will aid to elucidate the respective roles of Thr470 and Thr481 in PRC1-KIF4 complex formation and translocation during the metaphase-anaphase transition. In addition, molecular characterization of the phosphatase(s) responsible for the dephosphorylation of Thr470 and Thr481 will certainly better our understanding of the phospho-regulation cycle of PRC1 during mitosis.

Interestingly, the GFP-PRC1 ${ }^{\mathrm{T} 470 \mathrm{~A}}$-expressing telophase cells contain little cytoplasmic filaments while the midbody localization of the GFP signal remains (Figure 1C, $f^{\prime}$ ), yet there was no dramatic aneuploidy phenotype or severe cell cycle delay in these cells. One possibility is that endogenous PRC1 is sufficient to sustain cell cycle progression and cytokinesis in cells overexpressing $\mathrm{PRC} 1^{\mathrm{T} 470 \mathrm{~A}}$ given the fact that exogenous GFP-PRC1 level is only four-fold higher than that of endogenous PRC1. Since phosphorylation of Thr470 promotes microtubule bundling in vitro (Figure 3D), it is likely that cytoplasmic microtubule filaments are also stabilized by phosphorylated PRC1 in the telophase cells. Thus, examination of the precise spatiotemporal dynamics of Thr470 phosphorylation would be crucial to fully understand the molecular function and regulation of PRC1 in mitosis.

It has been shown that multiple microtubule-associated proteins act in concert to orchestrate cytokinesis. In anaphase cells, many of these proteins establish and/or associate with an anti-parallel central spindle microtubules. In addition to interacting with KIF4 to set up the central spindle, PRC1 also interacts with KIF14 and recruits it to the central spindle [5]. Further experiments will be required to address how multiple PRC1 protein complexes and PRC1 oligomers coordinate in a spatiotemporal manner in establishing and/or maintaining central spindle organization and dynamics.

\section{Acknowledgments}

We are grateful to Dr Wei Jiang for gift of reagents. We also thank the members of our group for helpful discussion during the course of this study. This work was supported by National Natural Science Foundation of China (39925018, 90508002 and 30121001), Chinese Academy of Science (KSCX1-R65 and RSCX2-H10), National Basic Research Program of China (973 project, 2002CB713700), American Cancer Society (RPG-99-173-01), a Gcc Breast Cancer Research award and National Institutes of Health grants DK56292 and CA89019 to XY (a GCC Eminent Scholar) and NS36194 (JW).

\section{References}

1 Guse A, Mishima M, Glotzer M. Phosphorylation of ZEN-4/ MKLP1 by aurora B regulates completion of cytokinesis. Curr Biol 2005; 15:778-786.

2 Neef R, Preisinger C, Sutcliffe J, et al. Phosphorylation of mitotic kinesin-like protein 2 by polo-like kinase 1 is required for cytokinesis. J Cell Biol 2003; 162:863-875.

3 Zhu C, Jiang W. Cell cycle-dependent translocation of PRC1 on the spindle by KIF4 is essential for midzone formation and cytokinesis. Proc Natl Acad Sci USA 2005; 102:343-348.

4 Kurasawa Y, Earnshaw WC, Mochizuki Y, Dohmae N, Todokoro K. Essential roles of KIF4 and its binding partner PRC1 in organized central spindle midzone formation. EMBO J 2004; 23:3237-3248.

5 Gruneberg U, Neef R, Li X, et al. KIF14 and citron kinase act together to promote efficient cytokinesis. J Cell Biol 2006; 172:363-372.

6 Mollinari C, Kleman JP, Jiang W, Schoehn G, Hunter T, Margolis RL. PRC1 is a microtubule binding and bundling protein essential to maintain the mitotic spindle midzone. J Cell Biol 2002; 157:1175-1186.

7 Mishima M, Kaitna S, Glotzer M. Central spindle assembly and cytokinesis require a kinesin-like protein/RhoGAP complex with microtubule bundling activity. Dev Cell 2002; 2:41-54.

8 Jiang W, Jimenez G, Wells NJ, et al. PRC1: a human mitotic spindle-associated CDK substrate protein required for cytokinesis. Mol Cell 1998; 2:877-885.

9 Ban R, Irino Y, Fukami K, Tanaka H. Human mitotic spindleassociated protein PRC1 inhibits MgcRacGAP activity toward Cdc42 during the metaphase. J Biol Chem 2004; 279:1639416402.

10 Mollinari C, Kleman JP, Saoudi Y, et al. Ablation of PRC1 by small interfering RNA demonstrates that cytokinetic abscission requires a central spindle bundle in mammalian cells, whereas completion of furrowing does not. Mol Biol Cell 2005; 16:10431055.

11 Zhu C, Lau E, Schwarzenbacher R, Bossy-Wetzel E, Jiang W. Spatiotemporal control of spindle midzone formation by PRC1 in human cells. Proc Natl Acad Sci USA 2006; 103:6196-6201.

12 Evans L, Mitchison TJ Kirschner MW. Influence of the centrosome on the structure of nucleated microtubules. J Cell Biol 1985; 100:1185-1191.

13 Glotzer M. The molecular requirements for cytokinesis. Science 2005; 307:1735-1739.

14 Mishima M, Pavicic V, Gruneberg U, Nigg EA, Glotzer M. Cell cycle regulation of central spindle assembly. Nature 2004; 430:908-913. 